\title{
Translocation of a granular chain in a horizontally vibrated saw-tooth channel
}

\author{
Fariba Mortazavi ${ }^{1}$, Mehdi Habibi ${ }^{1,2}$, a , and Ehsan Nedaaee Oskoee ${ }^{1, b}$ \\ 1 Institute for Advanced Studies in Basic Sciences, Gava Zang, Zanjan 45195-159, Iran \\ 2 Van der Waals-Zeeman Institute, University of Amsterdam, 1098 XH Amsterdam, The Netherlands
}

Received 24 January 2016 and Received in final form 21 July 2016

Published online: 21 October 2016

(C) The Author(s) 2016. This article is published with open access at Springerlink.com

\begin{abstract}
We study the translocation mechanism of a granular chain in a horizontally vibrated saw-tooth channel using MD simulations and macro-scale experiments and show that the translocation speed is independent of the chain length as long as the chain length is larger than the spatial period of the sawtooth. With the help of simulation, we explore the effect of geometry of the container and frequency and amplitude of vibration as well as chain flexibility on the chain drift speed. We observe that the most efficient transport is achieved when one of the channel walls is shifted with respect to the other wall by an amount equal to half the spatial period of the saw-tooth. We define a persistence length for the chain and show that the translocation speed depends on the ratio of persistence length over the spatial period of the saw-tooth. The optimum translocation occurs when this ratio is about 0.4. We also determine the optimum saw-tooth angle for the translocation of the chain as well as the optimum distance between the two walls. Some properties of this system are similar to those of polymer systems.
\end{abstract}

\section{Introduction}

A key step in viral infection is the transfer of viral DNA from viral capsid to the host cell through the tail tube. Therefore understanding this process is of great importance in biology and medical science. In recent years many theoretical and experimental studies have centered on DNA translocation and ejection [1-5]. For example, the effect of confinement on DNA mobility was characterized in slits and tube-like confinements [6,7] using micro/nanofluidic structures in weak and moderate confinement, allowing distinct regimes of transport and mobility of DNA molecules to be observed [8-10]. Despite these results, many aspects of the translocation of confined biopolymers are still unknown or subject of wide debate [11]. This is largely due to limitations in the available experimental techniques.

Brownian motors are essential tools in biology and nano-technology to extract useful work from a noisy environment. However, their governing principles are not limited to the nano scale and can also be implemented to macroscopic scales such as granular systems where a constant external driving force is provided by shaking the container. Most granular Brownian motors convert the random motion of the particles into a directed flow. This

\footnotetext{
a e-mail: m.habibi@uva.nl (corresponding author)

b e-mail: nedaaee@iasbs.ac.ir
}

can either be implemented by breaking the spatial symmetry of the boundary conditions [12-18] or by breaking the symmetry of the driving system [19-21]. In most examples of the first group, a saw-tooth-shaped boundary is used to break the symmetry $[12-18,22]$.

In ref. [23], Brownian dynamics simulations both in the absence and the presence of hydrodynamic interactions $[24,25]$ are used to study the dynamics of a polymer, subject to a ratchet potential. The average speed of the polymer and its conformational properties were examined upon variation in the polymer length, and the ratchet spatial period, where two distinct dynamical regimes were observed. In the regime of a small chain and long ratchet spatial periods, the velocity decreases when the chain length increases, while for long chains and small ratchet periods the velocity is insensitive to the variation of the chain length. One important application of such studies is in the transport and the size-based separation of biopolymers [26-28].

In recent years, the existence of athermal macroscopic systems that obey thermodynamic-like laws has been reported [29-33]. However, the application of thermodynamic/entropic concepts in macroscopic athermal systems is still a matter of open debate [33]. In these systems, due to the dissipative nature of the collisions, energy needs to be constantly supplied to keep the system moving. Therefore, the system is out of equilibrium. But as long as all possible states of the system can be properly sampled 
with equal probability, similar behavior can be expected for granular systems and their thermodynamic counterparts [32]. For granular systems, Edwards proposed a statistical approach to calculate the thermodynamic quantities from averages over grain configurations, but this approach was not established rigorously [29,34]. To explore the analogy between driven granular media and thermal equilibrium, Galanis et al. [32] studied a 2D binary mixture of granular particles (rods and spheres) and used entropy maximization to predict the dynamics. They showed that depletion interactions cause rods to form a specific configuration. Recently Windows-Yule et al. studied a 3D vibrated mixture of elongated athermal particles and applied the minimum free energy principle to explain the phase behavior and the ordered-disordered transition in this system. In this context, granular chains consisting of beads connected by flexible links and excited by vibration are introduced as a macroscopic model for polymer dynamics $[30,31,35,36]$. This macroscopic system is used to study unknotting, radius of gyration, ring diffusion, and entropic elasticity of chains [30,31,36]. A selfavoiding walk model for polymers was mimicked by performing experiments with a granular chain agitated by self-propelled balls [37]. Kudrolli and co-workers have also studied the structure and dynamics of vibrated granular chains [29] and diffusion of granular rods [38]. They compared their results for macroscopic chains with equilibrium polymer theories. Furthermore, studying the packing of granular chains in two and three-dimensions showed a close similarity to that in the glass transition of real polymers. In fact the glass transition in real polymers is equivalent to a jamming transition in granular chain systems $[39,40]$.

Interaction between granular chains and ratchet structures has also been studied. Chen et al. measured the force generated on a granular chain moving randomly on a periodic ratchet plate [41]. Furthermore, Lin and co-workers experimentally investigated the dynamics of a short granular chain on a one-dimensional gradient of vibration. They observed transitions from passive monotonic creeping against the gradient, to rapid stochastic head swinging with a reversed bias in its direction. Their experimental results for uniform vibrations showed a non-monotonic development of accessible modes behind the transitions, and confirmed a relationship between the size of the chain and the spatial gradient [42].

In this paper, we investigate the dynamics and translocation of a granular chain in a horizontally vibrated sawtooth channel. To study how breaking spatial symmetry by saw-tooth-shaped side walls of the narrow corridor leads to a drift speed on the chain, we introduce a molecular dynamics simulation method and simulate the translocation mechanisms of the chain. We also perform experiments in which a granular chain is shaken in a horizontal saw-tooth corridor. By direct imaging of the chain moving in the corridor we measure the drift speed and compare it with our simulation results. We explore the effect of geometry of the container as well as the flexibility and the length of the chain on the drift mechanism. The results of this work show strong similarities between

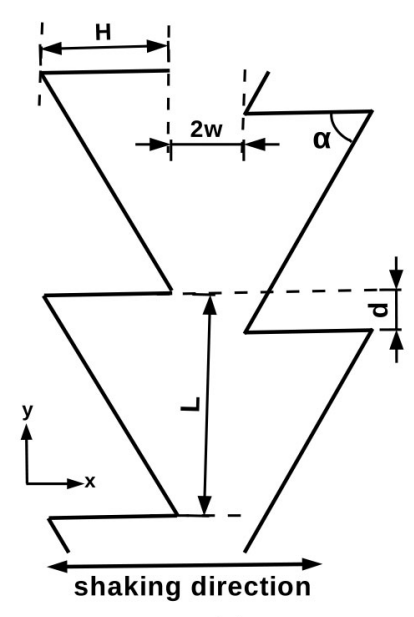

(a)

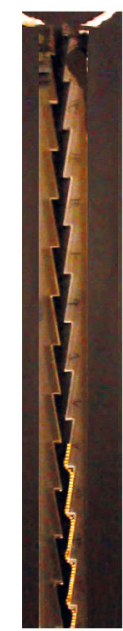

(b)

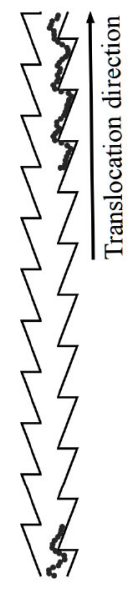

(c)
Fig. 1. a) The geometrical parameters of the system. b) Top view of the experimental setup. c) Visualization of the setup used in the simulations. The simulated chain re-enters the beginning of the channel as it reaches the end (periodic boundary condition). The vertical arrow shows the direction of the translocation while the horizontal arrows show the direction of vibration.

our out-of-equilibrium athermal system and its molecular counterparts and provide a new insight into the underlying entropic mechanisms of chain translocation under confinement which will be of wide interest to researchers in the field of granular materials and polymer physics.

\section{Methods}

The system studied here consists of a saw-tooth corridor in which one of the walls is shifted with respect to the other one as shown in fig. 1. The important geometrical parameters are shown in fig. 1a. $\alpha$ is the angle and $L$ is the spatial period of the saw-tooth, $H$ is the height of the teeth, $d$ is the amount of shift between the two walls and $w$ is half the distance between them as shown in fig. 1a. $\alpha$ can be set by $L$ and $H$. Therefore $(L, H, w, d)$ will give a set of independent geometrical parameters of the system. We have used the following values in our experiments and the majority of our simulations: $\alpha=80^{\circ}, L=4.3 \mathrm{~cm}$, $H=0.76 \mathrm{~cm}, d=L / 2$ and $w=0.35 \mathrm{~cm}$. When different sets of geometrical parameters are used in the simulation the corresponding values will be mentioned. The structure is shaken horizontally with the frequency $\omega$ in the $x$ direction.

The granular chain consists of spherical beads connected to each other by loose links. The length of the chain is described by the number of beads in the chain $(N)$. The maximum angle between the two consecutive links is $\theta_{\max }$ (fig. 2). We can define a persistence length $\left(l_{p}\right)$ for the chain by using the decay of the link-angle correlation: $\left\langle\cos \left(\theta_{n}-\theta_{1}\right)\right\rangle=\left\langle\cos \left(\theta_{2}-\theta_{1}\right)\right\rangle^{n-1} \sim \exp \left[-\frac{(n-1) D}{l_{p}}\right]$, where $\theta_{n}$ is the angle of the $n$-th link and $D$ is the diameter of 


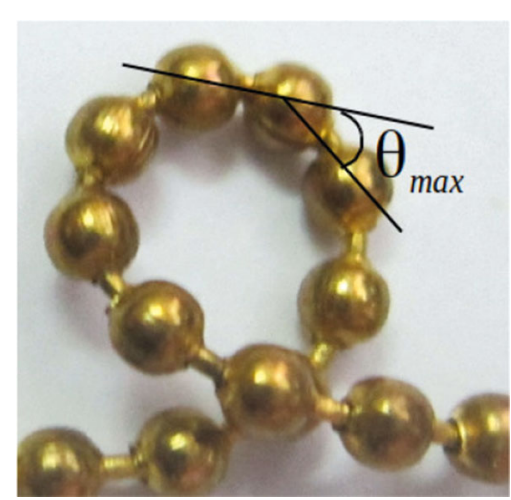

Fig. 2. $\theta_{\max }$ is the maximum angle between the two consecutive links and determines the rigidity of the chain. $\theta_{\max }$ and $l_{p}$ of the chain used in our experiments are $45^{\circ}$ and $21 \mathrm{~mm}$ respectively. The minimum number of beads required to form a ring is roughly the same as the persistence length of the chain.

the bead $[29,43]$. By assuming that $\left(\theta_{2}-\theta_{1}\right)$ is uniformly distributed between $-\theta_{\max }$ and $\theta_{\max }$, we can define the persistent length for a specific $\theta_{\max }$.

\subsection{Experimental procedure}

The experimental setup is composed of a fourteen-cell sawtooth corridor made from plexiglass shown in fig. 1b. The saw-tooth corridor is mounted horizontally and shaken in the $x$ direction using an off-center pulley which is driven by an AC motor [22]. The amplitude of the oscillations is chosen based on experimental observations to achieve a considerable interaction between the chain and the corridor walls. For very small oscillation amplitudes the chain does not interact with the corridor walls or gets stuck. In this work we limit ourselves to a shaking frequency of $4 \mathrm{~Hz}$ and an oscillation amplitude of $1.28 \pm 0.05 \mathrm{~cm}$ in both the experiments and the simulations, except for the data shown in figs. 7 and 8 in which the effects of the shaking frequency and oscillation amplitude are studied respectively.

The granular chain used in the experiments consists of hollow nickel-coated aluminium spherical beads of diameter $D=2.5 \pm 0.05 \mathrm{~mm}$ connected to each other by a loose link. The mass of one bead and its neighboring link is $m=0.033 \mathrm{gr}$. Two consecutive links are allowed to have a maximum angle of $\pi / 4 \mathrm{rad}$ and the distance between the two consecutive beads changes between 0 and $l=0.9 \mathrm{~mm}$. The persistence length of the chain calculated using the above method is about 9 times the diameter of the beads ( $\sim 21 \mathrm{~mm})$; the same as the estimation of ref. [29]. This is roughly the minimum number of beads required to form a ring (see fig. 2). This number can be used as an estimation to the rigidity of the chain $[29,41]$.

The length of the chain $(N)$ ranges from 5 to 156 in our experiments. The average drift speed of the chain is determined by taking direct images of the end point of the chain in time. The motion of the chain in the container is recorded by taking a movie using a CCD camera with a pixel resolution of $752 \times 582$ and frame rate of $24 \mathrm{~Hz}$.
The end position of the chain is determined to within a third of the bead diameter by analyzing the images. The $y$ coordinate of the end position is followed in time to obtain the drift speed. The average drift speed is finally calculated by taking an average over 12 runs.

The saw-tooth corridor is connected to a circular reservoir at the end of the corridor to collect the chains leaving the corridor. To avoid the influence of reservoir on the chain dynamics only those situations are considered here in which both ends of the chain are inside the corridor and the chain interacts only with the saw-tooth walls.

\subsection{Simulation method}

To elucidate the transport mechanism we perform molecular dynamics simulations. The net force imposed on each bead is a combination of normal and tangential forces, due to interactions with other beads as well as the container walls. The position of the centers of the beads $i$ and $j$ are given by $\vec{r}_{i}$ and $\vec{r}_{j}$. Hertzian formulation predicts that the normal force between particles is proportional to the normal compression to the power $3 / 2$ [44]. For viscoelastic particles a damping term need to be added to the elastic repulsion term $[45,46]$. Therefore the normal force between the beads $i$ and $j$ is given by

$$
\vec{F}_{n_{i, j}}=-f(\xi)\left(k D \xi-\gamma m_{\mathrm{eff}} v_{n_{i, j}}\right) \hat{n}_{i, j},
$$

where $k$ and $\gamma$ are the elastic and viscoelastic constant respectively, $v_{n_{i, j}}$ is the normal component of the relative velocity of different beads at the contact point, $\hat{n}_{i, j}=\frac{\vec{r}_{j}-\vec{r}_{i}}{\left|\vec{r}_{j}-\vec{r}_{i}\right|}$ is the normal unit vector at this point and $m_{\mathrm{eff}}=m_{i} m_{j} /\left(m_{i}+m_{j}\right)$ is the effective mass. Here, $\xi$ is the normal compression at the contact, calculated as $\xi=1-r_{i j} / D$, where $D$ is the bead diameter. According to Hertzian model $f(\xi)=\sqrt{\xi}$ for $\xi>0$ and $f(\xi)=0$ for non-positive $\xi[22,45,46]$.

Bead-bead collision - In general, for particles with different sizes and material properties, the coefficient of the elastic part is defined as

$$
k=\frac{4}{3} \sqrt{R_{\mathrm{eff}}}\left(\frac{1-\nu_{i}^{2}}{Y_{i}}+\frac{1-\nu_{j}^{2}}{Y_{j}}\right)^{-1},
$$

in which $R_{\text {eff }}^{-1}=R_{i}^{-1}+R_{j}^{-1}$ and $R_{i}, \nu_{i}$ and $Y_{i}$ are the radius, the Poisson ratio, and Young modulus of the grain $i$ respectively [46]. In fig. 3 the details of bead-bead collision are schematically shown. Elastic and viscoelastic constants for bead-bead interaction are shown with subscript "b-b".

Bead-wall collision - The same equations are used to model the interaction between the beads and the channel walls, in which the coefficients have an added subscript "b-w". Here we assume that the radius of curvature of the wall is infinity and the mass of the container is set to be infinity too. The viscoelastic tangential interaction 


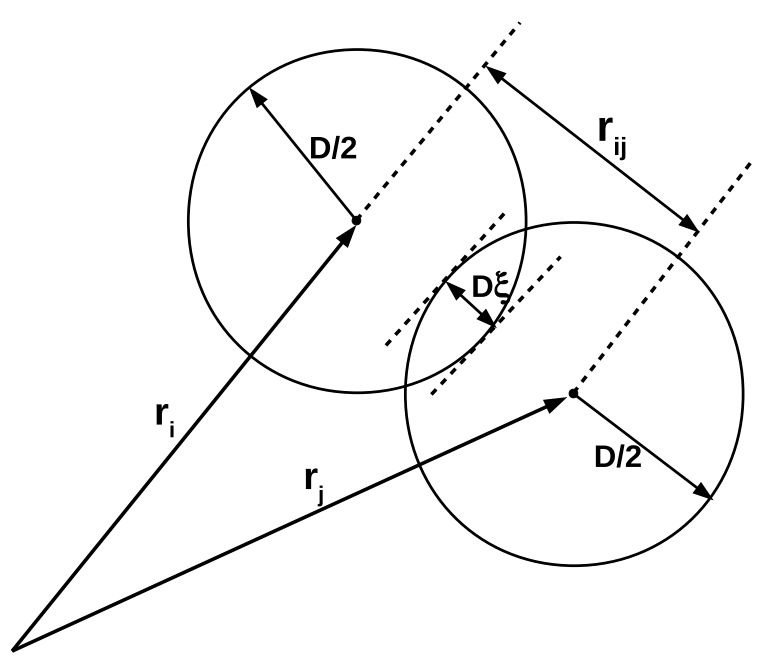

Fig. 3. The Hertzian model is used to simulate the contacts. The position of the centers of the beads $i$ and $j$ are given by $\vec{r}_{i}$ and $\vec{r}_{j} . \xi$ is the ratio of the normal compression at the contact as shown in the image.

between the beads and the tangential forces is neglected to assure that beads remain in contact with the floor of the channel [22].

Bead-bead bonding - In addition to the abovementioned repulsive interactions, there is an attractive interaction between the adjacent beads in the chain, and is applied when the distance between the center of mass of two adjacent beads exceeds the $D+l$ limit, where $l$ is the length of the loose rod. Equation (1) is employed to model this interaction with $f(\xi)=-\sqrt{\xi}$ for non-negative $\xi$ and $f=0$ otherwise. In this case, $\xi$ is defined as $\left\{r_{i j}-(D+l)\right\} / D$. The parameters of this attractive interaction are shown with subscript "att".

Bending rigidity - In order to take the bending rigidity of the chain into account, the Hertzian model (eq. (1)) with $\xi=\theta / \theta_{\max }-1$ is employed. Here $\hat{n}_{i j}$ is a unit vector perpendicular to the line passing from the center of adjacent beads, in a way to prevent bending. The numerical values of the coefficients of such interactions are shown with subscript "bend".

Calibration parameters - We consider the elastic and viscoelastic coefficients as calibration parameters by which one can adjust the simulation results to the experiments. All parameters in table 1, are only justified by their ability to reproduce the experimental results. We first started with a set of coefficients determined by numerically matching the experimental results of a bead bouncing off an aluminium and plexiglass plate for bead-bead and bead-wall interactions, respectively. Then we changed these values until we achieved the best possible match between the experimental values and simulation results of the drift speed.

Sliding criterion - To distinguish between sliding and rolling contacts a method proposed by Kondic [47] is used for evaluating the tangential force exerted on the chain
Table 1. The numerical values for the contact model coefficients. These parameters are chosen to achieve the best match to the experimental results. All values are given in the CGS system of units.

\begin{tabular}{cccc}
\hline \hline Coefficient & Numerical value & Coefficient & Numerical value \\
\hline$k_{\mathrm{b}-\mathrm{b}}$ & $2.187 \times 10^{5}$ & $\gamma_{\mathrm{att}}$ & 3240 \\
$k_{\mathrm{att}}$ & $2.187 \times 10^{5}$ & $\gamma_{\mathrm{b}-\mathrm{w}}$ & 255.75 \\
$k_{\mathrm{b}-\mathrm{w}}$ & $6.175 \times 10^{5}$ & $\gamma_{\text {bend }}$ & 27 \\
$k_{\text {bend }}$ & $5.38 \times 10^{4}$ & $\mu_{k}$ & 0.4 \\
$\gamma_{\mathrm{b}-\mathrm{b}}$ & 107.75 & $\mu_{s}$ & 0.5 \\
\hline \hline
\end{tabular}

by the channel surface. In a first step the contact is assumed to be rolling with $\mathbf{f}=m \mathbf{a}$. While the existence of loose links reduces the rotational degrees of freedom of the beads, one has to take the other degrees into account by computing the acceleration of the sphere as $\mathbf{a}=0.5 \mathbf{a}_{s}$, with $\mathbf{a}_{s}$ being the acceleration of the substrate. This is the final result if the no-sliding condition $\left(|\mathbf{f}| \leq f_{\max }=\mu_{s} m g\right)$ is satisfied. Otherwise, the sliding contact has to be evaluated using the dynamic friction coefficient $\mu_{d}$

$$
\mathbf{f}=\mu_{d} m g \frac{\mathbf{V}_{r s}}{\left|\mathbf{V}_{r s}\right|}
$$

where $\mathbf{V}_{r s}$ is the velocity of the contact point relative to the substrate.

Reference frame, boundary conditions and error bars Shaking is modeled by a sinusoidal excitation $x(t)=$ $x_{0} \cos (\omega t)$, where $\omega$ is the oscillation frequency and $x_{0}$ is its amplitude. Since all the simulations are performed in the referential frame of the container, a force $-\omega^{2} x_{0} \cos (\omega t)$, is added to each particles to convert noninertial frame of reference to inertial reference frame. The code is written in $\mathrm{C}++$ using a 5 th-order predictorcorrector algorithm for numerical integration of the equations of motion. The time step increment $(\Delta t)$ is set to $10^{-6} \mathrm{~s}$ in all simulations. The simulated system is composed of an eleven cell saw-tooth corridor as shown in fig. 1c. The simulation is run for a very long time with the periodic boundary condition, which means that the simulated chain re-enters the beginning of the corridor as it reaches the end (fig. 1c). Then the drift speed is calculated over different time intervals to estimate the error bars. The maximum error bar in our simulation is about $4 \%$ of the averaged value. So in most of the plots presented in this work the symbol size for the simulation data points are chosen to be larger than the error bars.

\section{Results and discussion}

\subsection{Experiment vs. simulation}

Performing experiments and simulations reveals that the chain is always transported in the corridor toward the wider part of the cells. The transport direction is shown in 


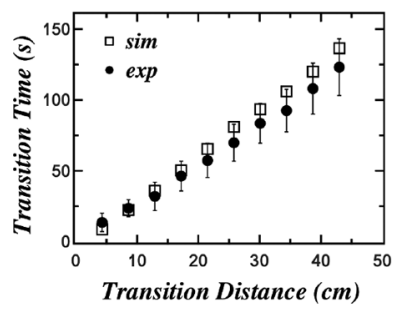

(a)

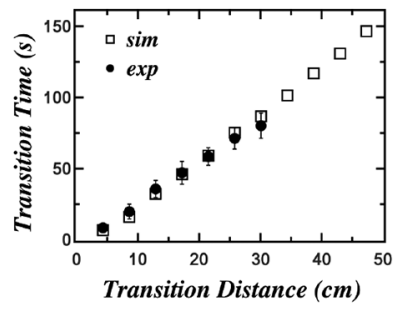

(c) (b)

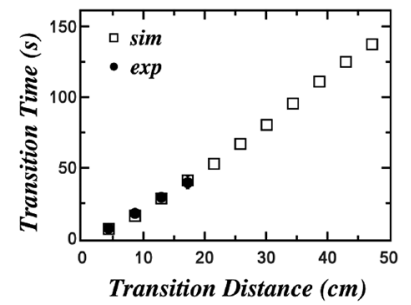

(d)

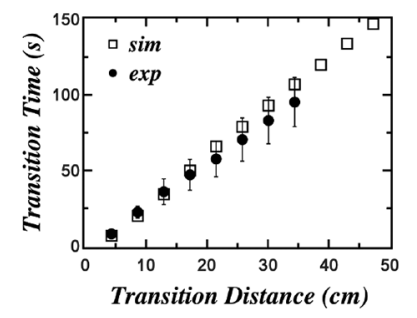

Fig. 4. The transition time of the head of the chain as a function of the transition distance for four different lengths of the chain measured in both experiments and simulations. The number of the beads in the chain are 41, 67, 94, and 134 for $\mathrm{a}, \mathrm{b}, \mathrm{c}$ and $\mathrm{d}$ respectively.

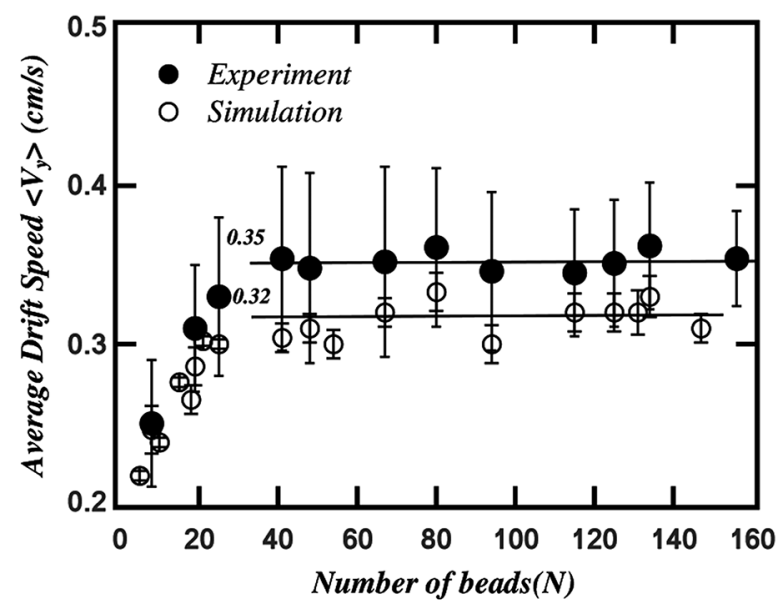

Fig. 5. Drift speed as a function of the length of the chain, for both experiment and simulation. Horizontal lines represent the average values for chains with $N>25$.

fig. 1c. Figure 4 shows the transition time of the head of the chain as a function of the transition distance for four different lengths of the chain. Both experimental (filled symbols) and simulation (open symbols) results for the measured time increase linearly as a function of the position which indicates that the average drift speed of the chain is constant in time. Linear fit to the data determines the drift speed of the chain for different lengths of the chain as shown in fig. 5 . Filled circles in fig. 5 represent the experimental value of the drift speed. The drift speed first increases with increasing the chain length for chain lengths below 25 beads and then reaches a plateau with an average speed of $0.35 \pm 0.03 \mathrm{~cm} / \mathrm{s}$ and $0.32 \pm 0.02 \mathrm{~cm} / \mathrm{s}$ for experiments and simulations, respectively. The drift speeds obtained by simulation are about $10 \%$ smaller than the results of our experiments but show the same trend. The plateaus in fig. 5 indicate that the drift speed is independent of the chain length except for the small chains. For chain lengths smaller than 25 beads, the end to end distance of the chain is comparable to or smaller than the spatial period of the saw-tooth and in most of the times the chain sticks in a single cell. But as long as the length of the corridor is large and the spatial period of the saw-tooth is small compared to the length of the chain, the length of the chain does not affect the translocation speed. For the majority of the data points shown in fig. 5, these two conditions are satisfied. Interestingly similar results are predicted for the simulation of DNA or polymers transported in the presence of a saw-tooth potential $[23,24,26,48]$ in which the drift speed of the polymer is shown to be insensitive to the length of the polymer.

\subsection{The transport mechanism}

In order to study the drift mechanism in detail, one needs to consider how the geometrical parameters of the channel or properties of the chain can affect the translocation. Performing experiments for all these cases is time consuming and sometimes impossible considering our experimental limitations. The fair agreement between the simulation results and experiments shown in fig. 5 indicates the validity of our simulation method. Therefore in the rest of the paper we present only the simulation results to gain insight into the driving mechanism of the chain translocation.

\section{i) Effect of chain rigidity on translocation}

Transport direction in our system is always toward the wider part of the cells. In fact the free ends of the chain prefer to move toward the wider parts of the cell which is entropically favorable as it has been shown before for the polymer systems $[49,50]$. The entropic origin of the transport indicates that the chain rigidity should influence the drift speed, as in polymer systems translocation is strongly affected by the persistence length of the polymer [51]. In our system the persistent length controls the chain's freedom to visit different parts of the cells. So we expect to hinder the drift speed of the chain by increasing its rigidity. The rigidity (flexibility) of the chain is defined by $\theta_{\max }$; the smaller the $\theta_{\max }$, the higher the rigidity of the chain. Although in our experiments $\theta_{\max }$ and consequently the persistence length are fixed, in our simulations we are able to change them and see how the rigidity affects the drift speed. In fig. 6 the drift speed of the chain is plotted as a function of the rescaled persistence length $\left(l_{p} / L\right)$, for five different lengths of the chain. The ratio of persistent length over the spatial period of the saw-tooth is the pertinent parameter control the structural entropy of the system. According to fig. 6 when $l_{p} / L \sim 0.4$ the data points are scattered around a drift speed of $0.3 \mathrm{~cm} / \mathrm{s}$. Here the chain is flexible and can easily be bent and form several loops. In this regime we have many structural configurations for the chain and consequently large areas of the 


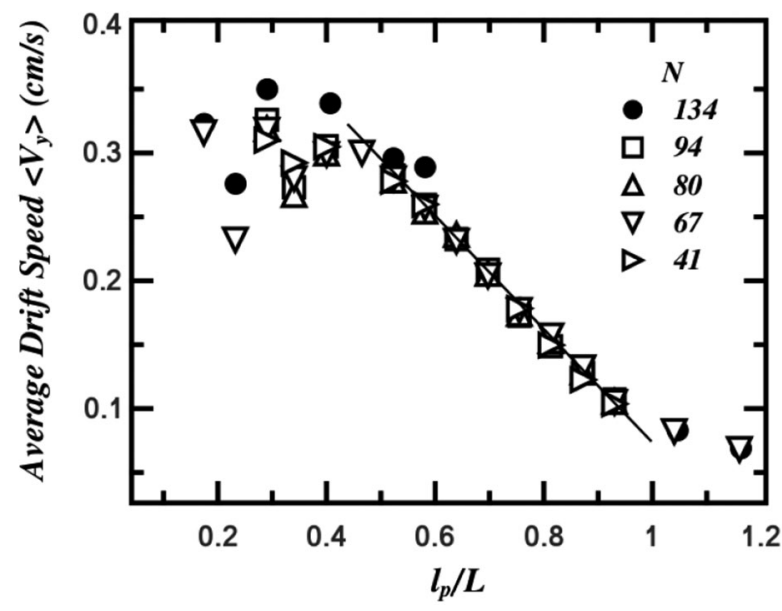

Fig. 6. Drift speed as a function of rescaled persistence length $\left(l_{p} / L\right)$ for different chain lengths.

cells will be visited by the chain. This causes a high drift speed. However for higher chain flexibilities $\left(l_{p} / L<0.4\right)$, the translocation speed slightly decreases mainly because the flexible chain gets stuck in the corners of the saw-tooth structure. For $\left(0.4<l_{p} / L<1\right)$ we also observe a decrease of the drift speed with increasing the rigidity of the chain as expected from the entropically-driven systems. By increasing the persistent length of the chain, possible structural configurations of the chain in the cell decrease considerably and when $l_{p}$ becomes close to $L$ the chain only forms sinusoidal patterns in the corridor with wavelengths of about $L$. In this regime all the data points for different chain lengths collapse on a single line at a slope of $-0.44 \mathrm{~cm} / \mathrm{s}$. Finally we arrive in the third regime when the persistent length is larger than the spatial period of the saw-tooth. Here the freedom of the chain is completely hindered and the drift speed slightly goes to zero by increasing the persistent length. The preceding simulation results on the ejection of the polymers from confinements predict faster ejections for flexible polymers [51], in qualitative agreement with our observations.

\section{ii) Role of oscillation frequency and amplitude}

The vibration of the saw-tooth channel is responsible for the chain motion; it pumps kinetic energy to the chain which in combination with the symmetry breaking due to the asymmetric geometry of the channel, produces a net drift speed in a direction perpendicular to the direction of vibration. Because of the periodic nature of the exerted force, the frequency of the vibration plays an important role in the transport mechanism. To investigate this, we have plotted the simulation predictions of the drift speed as a function of the oscillation frequency for a chain with 67 beads at a fixed oscillation amplitude of $1.28 \pm 0.05 \mathrm{~cm}$ (fig. 7). Simulation results of fig. 7 show that increasing the vibration frequency increases the drift speed. Our experiments reveal that the oscillation amplitude needs to be larger than about $1 \mathrm{~cm}$ to observe the drift mechanism in our experimental setup. Below this value the chain does not interact considerably with the corridor walls and gets

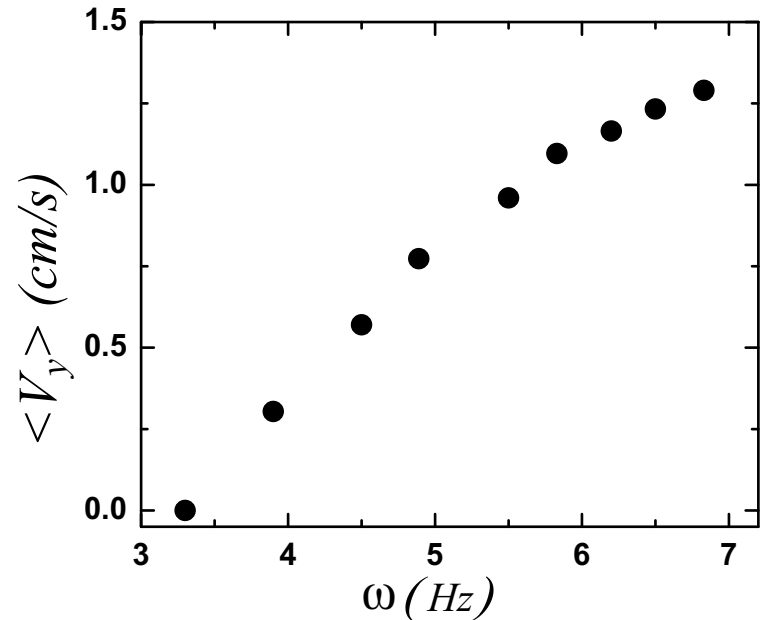

Fig. 7. Drift speed as a function of the vibration frequency, simulated for a chain of 67 beads. The drift speed increases with increasing the oscillation frequency.

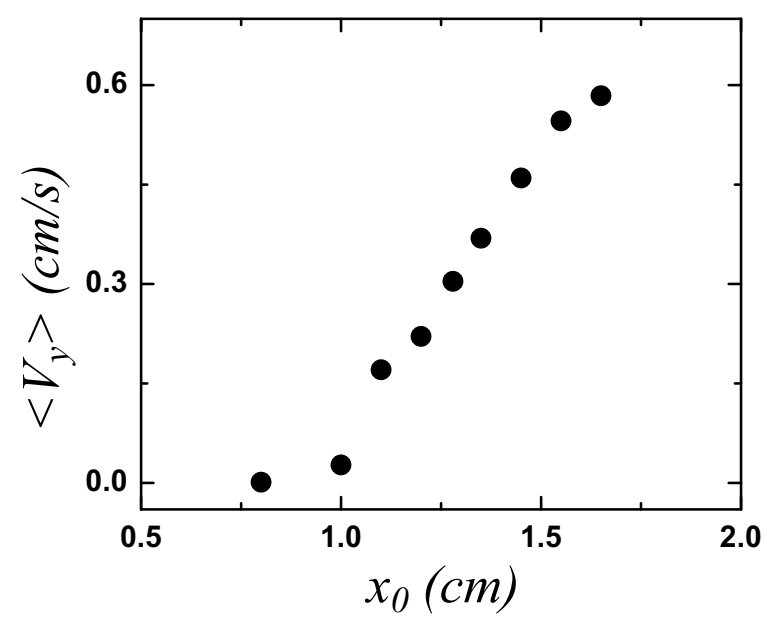

Fig. 8. Drift speed as a function of the oscillation amplitude for a chain of 67 beads. For amplitudes below $1 \mathrm{~cm}$ no translocation is observed. Drift speed shows an increasing trend with increasing the amplitude.

stuck. In fig. 8 the simulation results for the drift speed as a function of the oscillation amplitude are shown for a fixed oscillation frequency of $4 \mathrm{~Hz}$. These results indicate that for the oscillation amplitude below $1 \mathrm{~cm}$ the drift speed is close to zero in agreement with the experiments. The effect of the oscillation amplitude on the drift speed is similar to that of the oscillation frequency and causes an increase in the drift speed. To understand the effect of the oscillation parameters on the drift speed we can plot the drift speed as a function of the shaking acceleration $\left(\Gamma=x_{0}(2 \pi \omega)^{2}\right)$. In fig. 9 the dimensionless drift speed $\left(\left\langle v_{y}\right\rangle / v_{p}\right)$ is shown as a function of the dimensionless acceleration $(\Gamma / g)$ on logarithmic scale. The peak velocity of the container $\left(v_{p}=x_{0} /(2 \pi \omega)\right)$ and the gravitational acceleration $(g)$ are used to make the drift speed and the shaking acceleration dimensionless, respectively. Circles in 


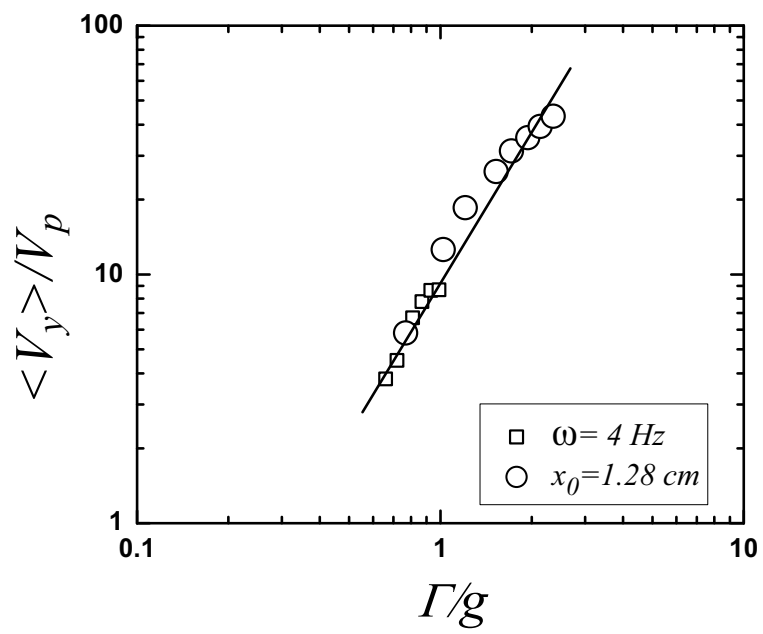

Fig. 9. Dimensionless drift speed $\left(\left\langle v_{y}\right\rangle / v_{p}\right)$ as a function of the dimensionless acceleration $(\Gamma / g)$ on Log-Log scale $(N=67)$. Circles represent the data of fig. 7 for a fixed amplitude of $1.28 \pm 0.05 \mathrm{~cm}$ and squares represent the data of fig. 8 for a fixed frequency of $4 \mathrm{~Hz}$. Both series collapse on a single line on Log-Log scale. The best power-law fit to all the data is $\left\langle v_{y}\right\rangle / v_{p}=9.89(\Gamma / g)^{1.96}$, shown by a solid line. The data points for amplitudes $0.8 \mathrm{~cm}$ and $1 \mathrm{~cm}$ in fig. 8 are not shown on this graph.

fig. 9 represent the data of fig. 7 for a fixed amplitude of $1.28 \pm 0.05 \mathrm{~cm}$ except for a single data point related to the smallest shaking frequency $(3.3 \mathrm{~Hz})$. The squares show the same data as those of fig. 8 for a fixed frequency of $4 \mathrm{~Hz}$ with the exception of the data points for amplitudes $0.8 \mathrm{~cm}$ and $1 \mathrm{~cm}$, for which the drift speed was close to zero. Both series of data collapse on a single line on LogLog scale with a slope of about 2 . The best power-law fit to all the data is shown by a solid line with an exponent of $1.96\left(\left\langle v_{y}\right\rangle / v_{p}=9.89(\Gamma / g)^{1.96}\right)$. According to fig. 9 the drift speed depends quadratically on the shaking acceleration $(\Gamma)$.

\section{iii) Effect of geometry on the transport mechanism}

The drift of the chain is a direct consequence of symmetry breaking imposed by the container geometry. To see how the geometry and the configuration of the boundaries affect the drift speed, the geometrical parameters describing the asymmetry of the channel have to be studied. Indeed, by introducing two parameters, one has a complete description of the asymmetric shape of the boundaries, i) the relative shifting of the two walls of the corridor, measured by the ratio $d / L$ and ii) the saw-tooth angle $\alpha$, as shown in fig. 1a. Changing these two parameters will change the shape and the geometry of the corridor and will affect the translocation as described in the following paragraph.

By considering the geometry of a corridor and neglecting the end boundaries we will see that a corridor with $d=A$ is a mirror image of one with $d=L-A$ as shown in fig. 10. This geometrical symmetry implies the same drift speed for both shifts and consequently a symmetric behavior for the drift speed around $d=L / 2$. Although in our experimental setup the parameter $d / L$ is fixed at $1 / 2$,

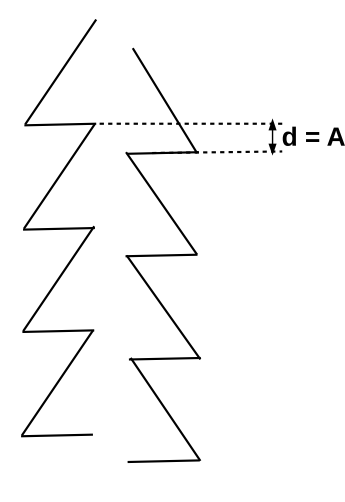

a

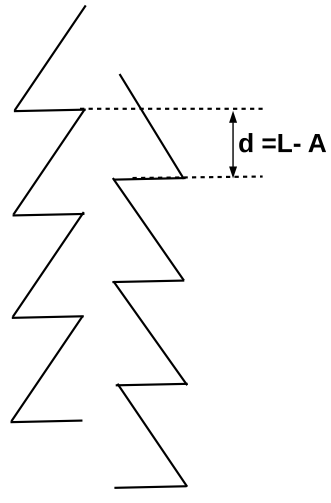

b
Fig. 10. A corridor with one of the walls shifted with $d=A$ (a) is a mirror image of one with $d=L-A$ (b).

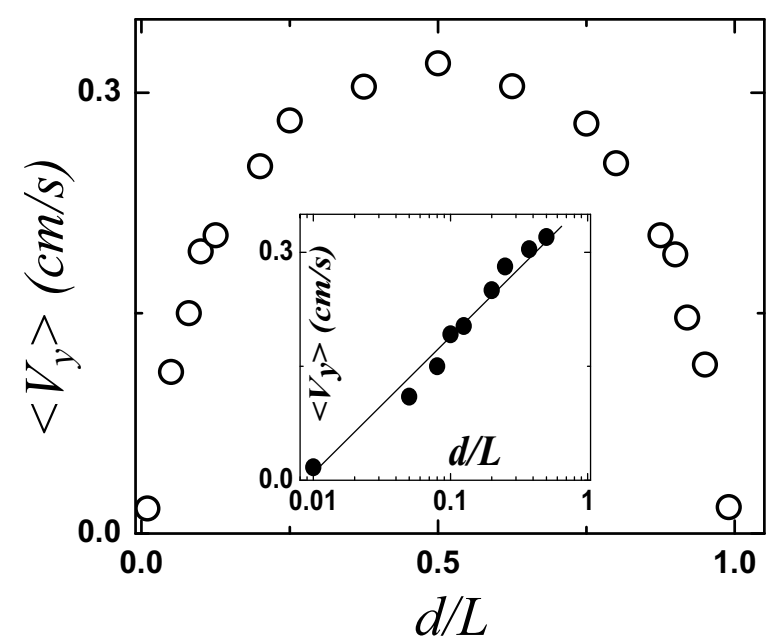

Fig. 11. Drift speed as a function of the shifting ratio $(d / L)$ for a chain of 67 beads. The data show a symmetric profile with a maximum at $d / L=1 / 2$. Inset: the same graph for $0<d / L<0.5$, on Lin-Log scale. The best logarithmic fit to the data is shown with a solid line $\left(\left\langle v_{y}\right\rangle=0.378+0.082 \operatorname{Ln}(d / L)\right)$.

in the simulation we can change the parameter $d$ from 0 to $L$. In fig. 11 the drift speed of the chain is shown as a function of $d / L$. The drift speed has a symmetric profile with a maximum speed at $d / L=0.5$ expected from the geometry of the corridor. This indicates that the most efficient transport is achieved when one of the walls is shifted by half the spatial period of the saw-tooth, as we had in our experimental setup. The same results are found for the transport of the granular beads in a similar system [22]. But in contrast with the granular bead system [22], which predicts a non-zero drift speed at $d=0$, our granular chain sticks in the corridor and does not move when the walls are not shifted.

The inset of fig. 11 shows the same data for $d / L \leq 0.5$ on Lin-Log scale with best logarithmic fit to the data $\left(\left\langle v_{y}\right\rangle=0.378+0.082 \operatorname{Ln}(d / L)\right)$. This graph shows that 


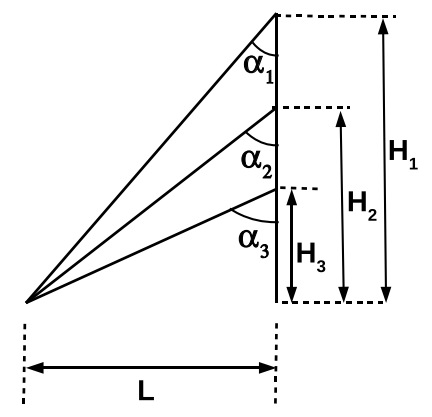

a

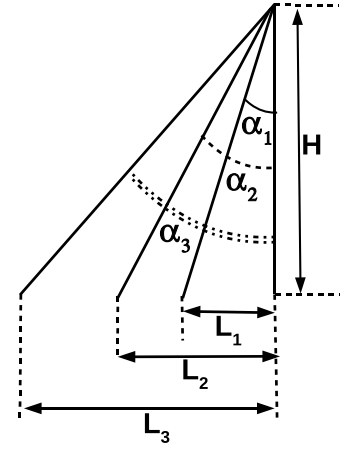

b
Fig. 12. a) $\alpha$ increases by decreasing $H$ while $L$ is fixed, b) $\alpha$ increases when $L$ increases and $H$ is fixed.

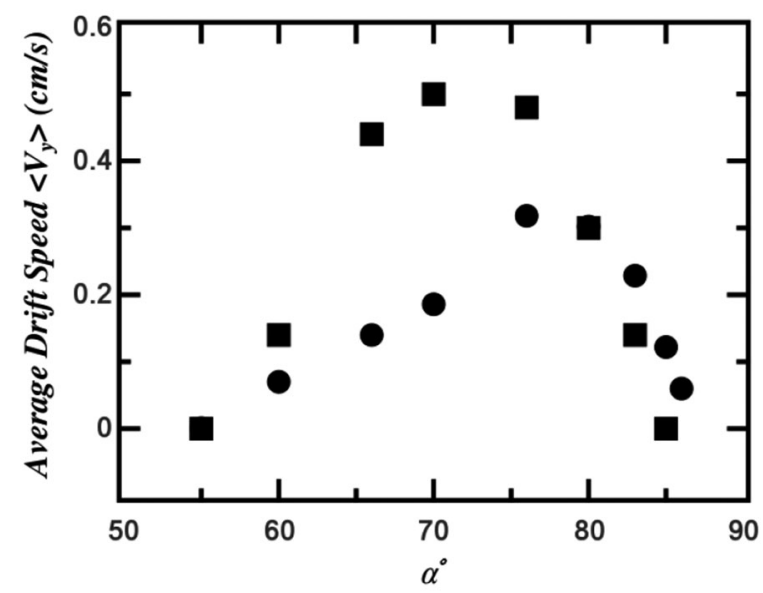

Fig. 13. Drift speed as a function of $\alpha$. Black squares represent the data for fixed $L$ and $H$ ranging from $0.38 \mathrm{~cm}$ to $3 \mathrm{~cm}$ while black dots show the data for fixed $H$ and $L$ changing from $1.3 \mathrm{~cm}$ to $10.86 \mathrm{~cm}$, for the following system parameters: $N=$ $67, d=L / 2$ and $w=0.35 \mathrm{~cm}$.

the drift speed changes with the amount of shift in a logarithmic manner.

The saw-tooth angle, $\alpha$, is another geometrical parameter that plays a great role in the transport mechanism. As it is schematically shown in fig. 12 , there are two ways of varying $\alpha$, i) $\alpha$ increases while $L$ is fixed and $H$ decreases (fig. 12a), ii) $\alpha$ increases while $H$ is fixed but $L$ increases (fig. 12b). In fig. 13 the drift speed is shown as a function of $\alpha$, when $\alpha$ is changing by both methods. For both cases $d=L / 2$. The squares represent a set of geometries in which $L$ is fixed at $4.3 \mathrm{~cm}$ but $H$ varies from $0.38 \mathrm{~cm}$ to $3 \mathrm{~cm}$. Here we observe an approximately symmetric profile for the drift speed as a function of $\alpha$ with a maximum around $\alpha=70^{\circ}$. But changing $\alpha$ using the second method with $H$ fixed at $0.76 \mathrm{~cm}$ and $L$ is varying from $1.3 \mathrm{~cm}$ to $10.86 \mathrm{~cm}$, gives an asymmetric profile for the drift speed with a maximum at $\alpha=76^{\circ}$. Furthermore the maximum drift speed obtained by the first method is larger than the second one.

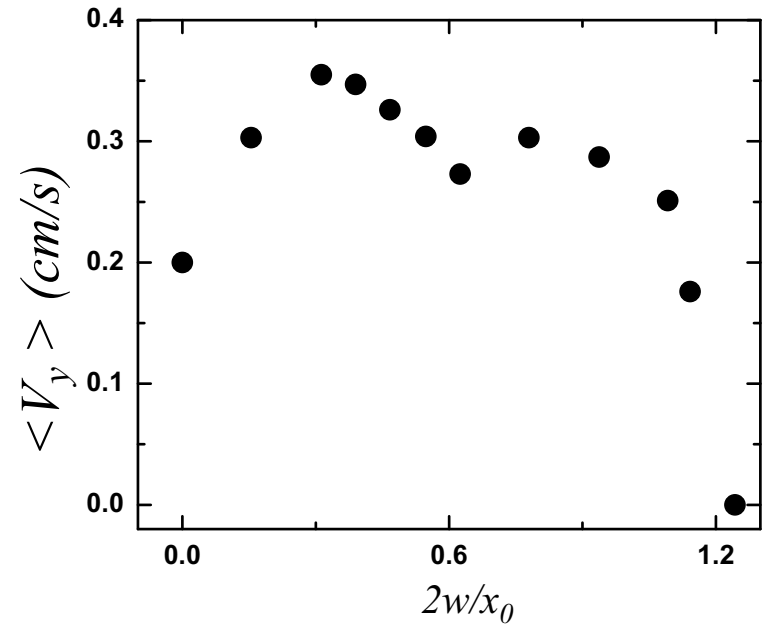

Fig. 14. Drift speed of the chain as a function of the distance between the two walls in a dimensionless form $\left(2 w / x_{0}\right)$ for $N=67, H=0.76 \mathrm{~cm}, L=4.3 \mathrm{~cm}$ and $d / L=1 / 2$.

Another important geometrical parameter in this system is the distance between the two walls of the corridor, $2 w$ (fig. 1a). Figure 14 shows the drift speed of the chain as a function of $2 w / x_{0}$. Here we scale the distance between the walls by the oscillation amplitude. The drift speed has a maximum at $2 w / x_{0} \sim 0.3$ and then decreases in a non-monotonic way. When the distance between the walls becomes larger than the amplitude $\left(2 w / x_{0} \gtrsim 1\right)$ the drift speed abruptly goes to zero as expected. In fact when the walls are far from each other with respect to the amplitude of the oscillation the interaction between the chain and both walls decreases, and results in decreasing the drift speed. On the other hand when the two walls are very close the chain will be stuck between the walls. Therefore there is an optimum value for the distance between the two walls where the speed is maximum.

Finally the drift speed changes with changing the spatial period of the saw-tooth when $H$ and $w$ are fixed. As observed in fig. 15, we find that the drift speed of the chain exhibits a single maximum upon variation with respect to $L$ at $L \sim 3 \mathrm{~cm}$. Data for different chain lengths in fig. 15 collapse on the same curve as long as $L$ is smaller than the chain length. This is another indication of the insensitivity of the drift speeds to the chain length, as discussed before.

\section{Conclusion}

In conclusion, we investigate the translocation of a granular chain due to spatial symmetry breaking in a horizontally vibrated saw-tooth corridor and study the effect of the geometry of the container, oscillation parameters as well as the flexibility of the chain on the drift mechanism and speed. We perform simulations and experiments of our system and justify our simulation results with the experiments. The transport always occurs toward the wider part of the cells due to entropically driven nature of the 


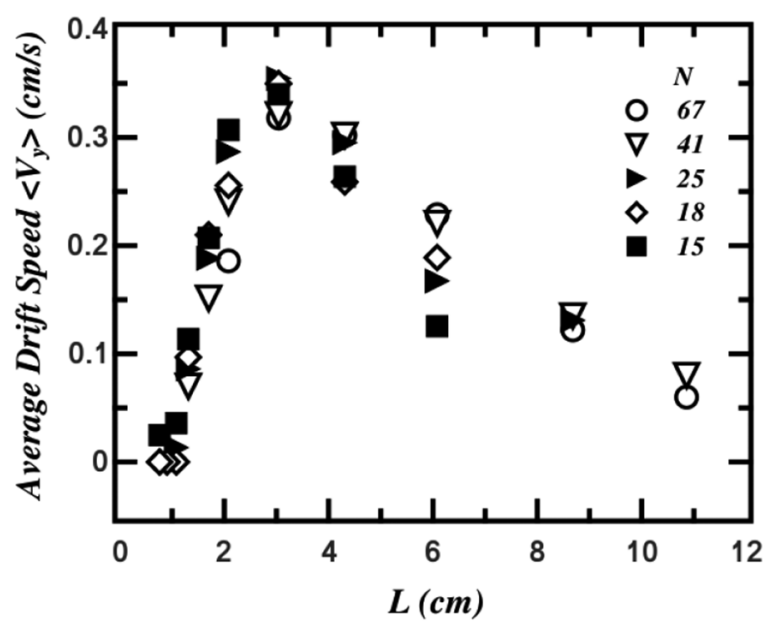

Fig. 15. Simulation results for the drift speed of the chain as a function of $L$ for different chain lengths and the following geometrical parameters: $d=L / 2, H=0.76 \mathrm{~cm}, l_{p}=1.2 \mathrm{~cm}$ and $w=0.35 \mathrm{~cm}$. The maximum drift speed occurs at $l_{p} / L \sim$ 0.4 .

problem. The molecular dynamics simulation enables us to explore the effect of different parameters such as flexibility of the chain, vibration frequency/amplitude, and geometry of the saw-tooth on the drift speed. In addition, we show that the important length scales are the persistence length of the chain and the spatial period of the saw-tooth. We find that as long as the chain length is larger than the spatial period of the structure the drift speed is independent of the chain length, similar to molecular counterparts of the system. The stiffness of the chain strongly affects the translocation speed. The maximum translocation speed occurs when the persistence length is about 0.4 of the spatial period of the saw-tooth. When $l_{p} / L$ is larger than 0.4 and below 1 , the translocation speed decreases linearly with increasing the persistent length of the chain independent of the chain length. The drift speed increases with increasing both the vibration frequency and the vibration amplitude and shows a quadratic relation with respect to the oscillation acceleration.

For geometrical parameters of the container, we predict an optimum saw-tooth angle for the translocation of the chain as well as an optimum distance between the two walls of the corridor. We show that the most efficient transport is achieved when one of the walls of the corridor is shifted an amount equal to half the spatial period of the saw-tooth, and the drift speed of the chain is increased logarithmically with the amount of the shift.

Similarities between our results and entropically driven polymeric systems indicate that the behavior of our outof-equilibrium system can obey the thermodynamic laws. Therefore understanding such macroscopic athermal systems may provide insights for studying more complicated systems e.g., DNA translocation where direct visualization is difficult. We believe these findings are of wide interest to researchers in the field of polymer physics, biophysics, and granular material. How entropy concepts can quantitatively describe the dynamics in our system is still unknown. Additional work is needed to understand the depth of this analogy and our findings may stimulate further work in this area.

\section{Author contribution statement}

MH designed the research. FM performed the simulations and the experiments under the supervision of ENO and MH. FM and ENO prepared the figures. All authors contributed to the analysis and interpretation of the results and writing the manuscript.

We thank Y. Rahmani for his help in preparing the experimental setup and H. Fazli for his useful discussions. We also thank B. Farnudi for his help in improving the writing of the manuscript. Support from the IASBS Research Council (Grant No. G2012IASBS103 and G2012IASBS106) is acknowledged. $\mathrm{MH}$ acknowledges the NWO-VIDI grant.

Open Access This is an open access article distributed under the terms of the Creative Commons Attribution License (http://creativecommons.org/licenses/by/4.0), which permits unrestricted use, distribution, and reproduction in any medium, provided the original work is properly cited.

\section{References}

1. M. Muthukumar, Phys. Rev. Lett. 86, 3188 (2001).

2. David Löf, Karin Schillén, Bengt Jönsson, Alex Evilevitch, J. Mol. Biol. 368, 55 (2007).

3. Davide Marenduzzo, Cristian Micheletti, Enzo Orlandini, J. Phys. Condens. Matter 22, 283102 (2010).

4. Marta De Frutos, Lucienne Letellier, Eric Raspaud, Biophys. J. 88, 1364 (2005).

5. Martin Castelnovo, Alex Evilevitch, Eur. Phys. J. E 24, 9 (2007).

6. Derek Stein, Frank H.J. van der Heyden, Wiepke J.A. Koopmans, Cees Dekker, Proc. Natl. Acad. Sci. U.S.A. 103, 15853 (2006).

7. Joshua David Cross, Elizabeth A. Strychalski, H.G. Craighead, J. Appl. Phys. 102, 4701 (2007).

8. Jonas O. Tegenfeldt, Christelle Prinz, Han Cao, Steven Chou, Walter W. Reisner, Robert Riehn, Yan Mei Wang, Edward C. Cox, James C. Sturm, Pascal Silberzan et al., Proc. Natl. Acad. Sci. U.S.A. 101, 10979 (2004).

9. Walter Reisner, Keith J. Morton, Robert Riehn, Yan Mei Wang, Zhaoning Yu, Michael Rosen, James C. Sturm, Stephen Y. Chou, Erwin Frey, Robert H. Austin, Phys. Rev. Lett. 94, 196101 (2005).

10. Fredrik Persson, Pawel Utko, Walter Reisner, Niels B. Larsen, Anders Kristensen, Nano Lett. 9, 1382 (2009).

11. Paul Grayson, Lin Han, Tabita Winther, Rob Phillips, Proc. Natl. Acad. Sci. U.S.A. 104, 14652 (2007).

12. I. Derényi, P. Tegzes, T. Vicsek, Chaos 8, 657 (1998). 
13. Z. Farkas, P. Tegzes, A. Vukics, T. Vicsek, Phys. Rev. E 60, $7022(1999)$

14. M. Levanon, D.C. Rapaport, Phys. Rev. E 64, 011304 (2001).

15. Zénó Farkas, Ferenc Szalai, Dietrich E. Wolf, Tamás Vicsek, Phys. Rev. E 65, 022301 (2002).

16. J.F. Wambaugh, Charles Reichhardt, C.J. Olson, Phys. Rev. E 65, 031308 (2002).

17. A.J. Bae, W.A.M. Morgado, J.J.P. Veerman, G.L. Vasconcelos, Physica A 342, 22 (2004).

18. Michael Heckel, Patric Müller, Thorsten Pöschel, Jason A.C. Gallas, Phys. Rev. E 86, 061310 (2012).

19. J.A.C. Gallas, H.J. Herrmann, S. Sokołowski, Physica A 189, 437 (1992).

20. R. Grochowski, P. Walzel, M. Rouijaa, C.A. Kruelle, I. Rehberg, Appl. Phys. Lett. 84, 1019 (2004).

21. Johannes Blaschke, Jürgen Vollmer, Phys. Rev. E 87, 040201 (2013).

22. Shahin Mobarakabadi, Ehsan Nedaaee Oskoee, Matthias Schröter, Mehdi Habibi, Phys. Rev. E 88, 042201 (2013).

23. James M. Polson, Brian Bylhouwer, Martin J. Zuckermann, Arthur J. Horton, William M. Scott, Phys. Rev. E 82, 051931 (2010).

24. Matthew T. Downton, Martin J. Zuckermann, Erin M. Craig, Michael Plischke, Heiner Linke, Phys. Rev. E 73, 011909 (2006).

25. Martin Kenward, Gary W. Slater, Phys. Rev. E 78, 051806 (2008).

26. Joel S. Bader, Richard W. Hammond, Steven A. Henck, Michael W. Deem, Gregory A. McDermott, James M. Bustillo, John W. Simpson, Gregory T. Mulhern, Jonathan M. Rothberg, Proc. Natl. Acad. Sci. U.S.A. 96, 13165 (1999).

27. Steven A. Henck, Michael W. Deem, Gregory A. McDermott, James M. Bustillo, Jonathan M. Rothberg, Electrophoresis 21, 74 (2000).

28. J.S. Bader, M.W. Deem, R.W. Hammond, S.A. Henck, J.W. Simpson, J.M. Rothberg, Appl. Phys. A 75, 275 (2002).

29. Kevin Safford, Yacov Kantor, Mehran Kardar, Arshad Kudrolli, Phys. Rev. E 79, 061304 (2009).
30. Z.A. Daya, E. Ben-Naim, R.E. Ecke, Eur. Phys. J. E 21 , $1(2006)$.

31. M.B. Hastings, Z.A. Daya, E. Ben-Naim, R.E. Ecke, Phys. Rev. E 66, 025102 (2002).

32. J. Galanis, R. Nossal, D. Harries, Soft Matter 6, 1026 (2010).

33. C.R.K. Windows-Yule, B.J. Scheper, W.K. den Otter, D.J. Parker, A.R. Thornton, Phys. Rev. E 93, 020901 (2016).

34. S.F. Edwardes, Granular Matter: An Interdisciplinary Approach, edited by A. Mehta (Springer, New York, 1994).

35. T.A. Witten, Rev. Mod. Phys. 70, 1531 (1998).

36. Eli Ben-Naim, Z.A. Daya, Peter Vorobieff, Robert E. Ecke, Phys. Rev. Lett. 86, 1414 (2001).

37. Jeffrey J. Prentis, Daniel R. Sisan, Phys. Rev. E 65, 031306 (2002).

38. V. Yadav, A. Kudrolli, Eur. Phys. J. E 35, 104 (2012).

39. Ping-Ping Wen, Ning Zheng, Liang-Sheng Li, Heng Li, Gang Sun, Qing-Fan Shi, Phys. Rev. E 85, 031301 (2012).

40. Ling-Nan Zou, Xiang Cheng, Mark L. Rivers, Heinrich M. Jaeger, Sidney R. Nagel, Science 326, 408 (2009).

41. KuanHua Chen, Y.C. Chou, Kiwing To, Phys. Rev. E 87, 012711 (2013).

42. W.-T. Lin, Y.-C. Sun, C.-C. Chang, Y.-C. Lin, C.-W. Peng, W.-T. Juan, J.-C. Tsai, Phys. Rev. Lett. 112, 058001 (2014).

43. Masao Doi, Sam F. Edwards, The Theory of Polymer Dynamics, Vol. 73 (Oxford University Press, 1988).

44. H. Hertz, J. Reine Angew. Math. 92, 156 (1881).

45. Nikolai V. Brilliantov, Frank Spahn, Jan-Martin Hertzsch, Thorsten Pöschel, Phys. Rev. E 53, 5382 (1996).

46. Thorsten Pöschel, Thomas Schwager, Computational Granular Dynamics: Models and Algorithms (Springer Science \& Business Media, 2005).

47. Ljubinko Kondic, Phys. Rev. E 60, 751 (1999).

48. Andrew Belmonte, Michael J. Shelley, Shaden T. Eldakar, Chris H. Wiggins, Phys. Rev. Lett. 87, 114301 (2001).

49. Narges Nikoofard, Hamidreza Khalilian, Hossein Fazli, J. Chem. Phys. 139, 074901 (2013).

50. Sarah E. Henrickson, Martin Misakian, Baldwin Robertson, John J. Kasianowicz, Phys. Rev. Lett. 85, 3057 (2000).

51. I. Ali, D. Marenduzzo, J.M. Yeomans, Phys. Rev. Lett. 96, 208102 (2006). 